\title{
Bedside Patent Ductus Arteriosus Ligation in Premature Infants
}

\author{
Basak Soran Turkcan¹, Atakan Atalay², Davut Bozkaya ${ }^{3}$, Emre Kulahcioglu² and Irfan Tasoglu² \\ ${ }^{1}$ Department of Pediatric Cardiovascular Surgery, Gaziantep Cengiz Gokcek Maternal Health and Children Hospital, Gaziantep, Turkey \\ ${ }^{2}$ Department of Pediatric Cardiovascular Surgery, Ankara City Hospital, Ankara, Turkey \\ ${ }^{3}$ Department of Neonatology, Ankara City Hospital, Ankara, Turkey
}

\begin{abstract}
Objective: To determine the safety of on-site bedside patent ductus arteriosus (PDA) ligation, performed by a regional roving surgical team at different neonatal intensive care units (NICUs) in preterm infants with hemodynamically significant PDA (hsPDA).

Study Design: A descriptive study.

Place and Duration of Study: Department of Paediatric Cardiovascular Surgery, Ankara City Hospital, Ankara, Turkey, between January 2018 and December 2020.

Methodology: Medical data of 48 premature infants with hsPDA, who underwent bedside PDA ligation by the same roving surgical team at five different NICU centres in Ankara province, were evaluated. Demographic and clinical data of the patients were extracted from the institutional databases and medical records. Postoperative complications were recorded.

Results: Mean body-weight of the infants was $1113.1 \pm 392.8$ grams. Forty patients were under respiratory support before surgery. Majority of the patients $(n=45,93.7 \%)$ no longer needed respiratory support after the third and seventh days of surgery. No complications related to surgery or anaestesia were observed. Non of the patients developed post-PDA ligation cardiac syndrome. No surgical wound infection or sepsis was observed in any patient. The overall mortality rate was $4.2 \%$.

Conclusion: Bedside PDA ligation at different NICUs by the roving surgical team is safe and effective. Owing to an extremely low risk, this procedure can be performed successfully in the hands of professional and experienced surgeons. It seems to be a feasible option for premature infants with hsPDA, who do not respond to medical treatment.
\end{abstract}

Key Words: Infant, Patent ductus arteriosus, Ligation, Intensive care unit, Newborn, Surgery.

How to cite this article: Turkcan BS, Atalay A, Bozkaya D, Kulahcioglu E, Tasoglu I. Bedside Patent Ductus Arteriosus Ligation in Premature Infants. J Coll Physicians Surg Pak 2022; 32(02):208-212.

\section{INTRODUCTION}

Patent ductus arteriosus (PDA) is one of the most common congenital heart defects in newborns, particularly in premature infants born before the 28th gestational week. ${ }^{1}$ The incidence of PDAis inversely related to the gestational age and weight. Although the maintenance of ductal patency is essential for the normal development of the fetus, persistent patency of the ductus arteriosus is associated with significant morbidity and mortality. In persistent PDA, left-to-right shunting of blood increases the pulmonary blood flow in infants; thereby, resulting an increased rate of morbidities and mortality due to stealing blood from the systemic circulation. ${ }^{2,3}$ Complications associated with prolonged haemodynamically significant PDA (hsPDA) consist of pulmonary oedema, congestive heart failure, ventilator dependence, bronchopulmonary dysplasia, necrotizing enterocolitis and death. ${ }^{4}$

Correspondence to: Dr. Basak Soran Turkcan, Department of Pediatric Cardiovascular Surgery, Gaziantep Cengiz Gokcek Maternal Health and Children Hospital, Gaziantep, Turkey

E-mail: basaksoran@gmail.com

Received: January 03, 2021; Revised: May 31, 2021;

Accepted: June 19, 2021

DOI: https://doi.org/10.29271/jcpsp.2022.02.208
Over the past three decades, there has been a pradigm shift in presentation and treatment of infants with PDA. Before the use of antenatal corticosteroids, PDA wasfrequently found in premature infants of all gestational ages, and was associated with respiratory distress syndrome. Indomethacin was standard treatment; and the studies mostly evaluated the benefits of early (or even prophylactic) PDA ligation. With recent advances in ventilation strategies, the use of antenatal corticosteroids and exogenous surfactant, and increased willingness to wait for spontaneous ductus arteriosus closure, more mature preterm infants rarely require intervention for a ductus arteriosus, currently. Instead, PDA management is focused on the most premature infants in whom the ductus can be resistant to pharmacological treatment. ${ }^{5}$

Transporting infants to a referral centre for the preoperative period is a widespread practice; however, as an alternative which minimises the risk of neonatal transfer, a roving operative team may also provide bedside ligation in the home setting. The main advantages of bedside PDA closure include avoidance of hypothermia, continuation of preoperative high-frequency ventilation and reduced risk of line dislodgement and unplanned extubation. Several retrospective studies have reported safely performing bedside PDA ligation with surgical 
outcomes comparable to operating room closure, low rates of postoperative infection and reduced resource utilisation. Before considering bedside surgical ligation, a multidisciplinary discussion ensuring adequate resources including personnel, equipment and ambient conditions, is recommended.

Although bedside ligation in neonatal intensive care units (NICUs) may be a good option, surgical ligation is avoided due to the controversy of the safety and effectiveness of this procedure in premature infants.

The aim of the present study was to determine the safety and effectivesness of on-site bedside PDA ligation performed by a regional roving surgical team in different NICUs in prrmature infants with hsPDA.

\section{METHODOLOGY}

This descriptive study was conducted at five different NICU centres in Ankara province of Turkey between January 2018 and December 2020. A total of 48 premature infants (gestational age $<35$ weeks and birth weight $<2500 \mathrm{~g}$ ) with hsPDA, who underwent bedside PDA ligation by the same surgical team, were included in the study. The roving team, consisted of paediatric cardiovascular surgeons of Ankara City Hospital, performed PDA ligation in the NICUs of five different hospitals in the home setting. The decisions for surgical ligations were given by a paediatric heartteam consisting of neonatologists, paediatric cardiologists, and paediatric cardiovascular surgeons. All patients previously received medical treatment for hsPDA (two or three times), and the decision for surgical intervention was made when unresponsiveness to treatment was documented. Diagnostic criteria of hsPDA were the combination of clinical and echocardiography findings, while making a decision to proceed with surgical ligation. Respiratory symptoms were defined as ventilator dependence, associated with cardiomegaly and pulmonary oedema on chest radiography. Evidence of post-ductal end-organ compromise was defined as elevated creatinine, metabolic acidosis, and abdominal distension with ischemic ileus. A ductal diameter of $>1.5 \mathrm{~mm}$ on echocardiography was used as the threshold for determining early pharmacological treatment, while large ductal shunts were significant when the ductal diameter is $>2$ $\mathrm{mm}$. Among ventilator-dependent extremely preterm infants with persistent PDA, a larger ductal diameter $(>2.5 \mathrm{~mm})$ and severe echocardiography indices of the left heart volume loading were considered to be a shorter time to successful extubation, providing evidence of association between ductal shunt volume and benefit of surgical closure.,

Data including postnatal age and bodyweight at the time of the operation, PDA diameter, need for inotropic support, amount of chest tube drainage, duration of chest tube drainage, incidence of pneumothorax, duration of mechanical ventilation, incidence of surgical site infection, and mortality rate were retrieved from the institutional databases and medical records. Prior to study, a written informed consent was obtained from parents and/or legal guardians of the patients. The study protocol was approved by the Institutional Ethics Committee. (No: E1-20-1408, Date: 23/12/2020). The study was conducted in accordance with the principles of the Declaration of Helsinki.

Under short term general anaesthesia, PDA ligation was performed bedside inthe NICUsetting. Preoperative premedication and vital signs during surgery were monitored by the neonatologist. The length of the incision was approximately 2 to $4 \mathrm{~cm}$ via a left posterolateral thoracotomy, which was sufficient for comfortably exposing the surgical site. The aorta was carefully freed following lung retraction after thoracotomy; and PDA was meticulously dissected. Subsequently, PDA was clipped with two clips after the clip holder controlled outside the surgical field to avoid undeserible injuries (Figure 1). After ligation, the intercostal drainage tube was placed into the left pleural space. Lung expansion of the patients was examined through daily chest- $X$ rays; and drainages were recorded on an hourly basis. The chest tube was removed after full pulmonary expansion was ensured and confirmation of no visible air leak. Following surgery, all patients were followed in the same NICU until discharge.

Statistical analyses were performed using the SPSS for Windows version 15.0 software (SPSS Inc, Chicago, IL, USA). Continuous variables were expressed in mean \pm standart deviation (SD) or median (min-max), while categorical variables were expressed in numbers and percentage.

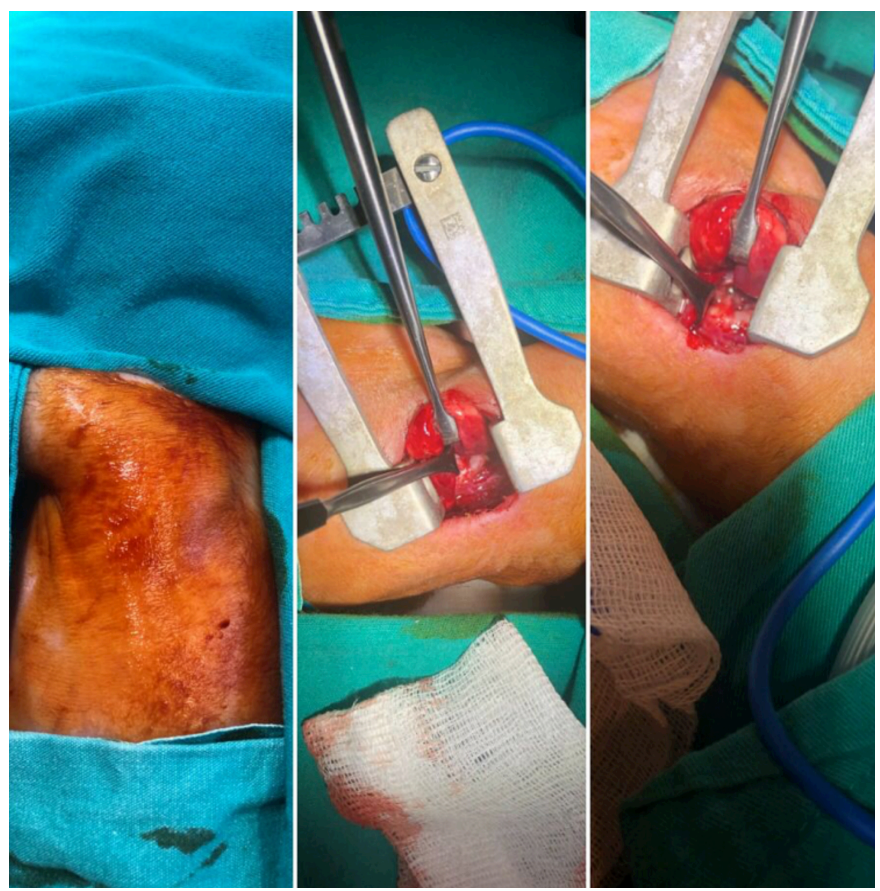

Figure 1: Intraoperative images of a bedside PDAclosure.

\section{RESULTS}

Bedside PDA ligations were performed in 48 premature infants in five different NICU centres during the study period. Before surgery, 36 (75\%) patients were intubated and 12 (25\%) patients were on nasal synchronised intermittent mandatory ventilation. Demographics of the patients, postoperative outcomes, and body weight at the time of operation are summarised in Table I. 
Table I: Demographics of the patients, postoperative outcomes, and body weight at the time of operation.

\begin{tabular}{|l|l|}
\hline Variables & $\mathbf{N}=\mathbf{4 8}$ \\
\hline Postnatal age (days) & $35.5 \pm 17.1$ (min-max) \\
\hline Body weight at operation (g) & $1113.1 \pm 392.8$ (min-max) \\
\hline PDA diameter (mm) & $2.3 \pm 0.6$ (min-max) \\
\hline $\begin{array}{l}\text { Duration of postoperative } \\
\text { mechanical ventilation (hours) }\end{array}$ & $74.1 \pm 41.7$ (min-max) \\
\hline $\begin{array}{l}\text { Sex }(\mathrm{n}, \%) \\
\begin{array}{l}\text { Female } \\
\text { Male }\end{array}\end{array}$ & $20(41.7 \%)$ \\
\hline Low birth weight $(<2500 \mathrm{~g})(\mathrm{n}, \%)$ & $7(14.6 \%)$ \\
\hline $\begin{array}{l}\text { Very low birth weight }(<1500 \mathrm{~g}) \\
(\mathrm{n}, \%)\end{array}$ & $16(33.3 \%)$ \\
\hline $\begin{array}{l}\text { Extremely low birth weight }(<1000 \mathrm{~g}) \\
(\mathrm{n}, \%)\end{array}$ & $25(52.1 \%)$ \\
\hline
\end{tabular}

Haemodynamic parameters remained stable during and after the operation. The mean operation time was $33.5 \pm 4.4$ (range, 25 to 44) min. No anaesthesia-related complications were seen during the operation. After postoperative days 3 to 7, 45 (93.7\%) patients did notneed respiratory support. Operation-related early complications, such as death, bleeding, pneumothorax, chylothorax, recurrent laryngeal nerve injury, or iatrogenic ligation of the aorta were not observed. None of the patients developed post-PDA ligation cardiac syndrome. The 24-hour chest tube drainage volume was often immeasurably low and usually less than $5 \mathrm{~mL}$ in total.

No surgical wound infection or surgery-related sepsis was observed inany patient during the follow-up period. Non-haemodynamically significant residual PDA was detected in two patients. During the follow-up period, residual PDA was spontaneously closed in both patients. Two (one with multiple congenital anomalies) of 48 patients (4.2\%) died of non-cardiac problems several days after the operation. One of the patients died after 14 days from non-cardiac congenital anomalies. The other patient died 70 days after operation from aspiration during bottle feeding.

\section{DISCUSSION}

Patent ductus arteriosus is a medical condition which causes serious hemodynamic, pulmonary, gastrointestinal, cerebrovascular, and retinal problems in infants hospitalised in NICU. It may be treated medically or surgically; however, no consensus has been established which method is the most optimal. The indications for surgical ligations arise in cases in whom indomethacin, ibuprofen, and paracetamol treatments fail or are contraindicated. ${ }^{7}$ An expectative approach after treatment failure was followed by spontaneous closure in $67-86 \%$ of the patients. ${ }^{6}$ In addition, the timing of surgical ligation of PDA in preterm infants still remains controversial. Prolonged exposure to ductus arteriosus shunting, a longer duration of pulmonary hyperemia and systemic hypoperfusion, may cause worse lung conditions, and lead to poorer nutritional status in late ligation. A recent meta-analysis showed that, compared to late surgical ligation, early ligation (at two or three weeks of life) might have a better respiratory outcome and nutritional status for PDA in preterm orvery low body weight (VLBW) infants. ${ }^{4}$
The response rates to medical treatment are lower in patients with VLBW. ${ }^{8}$ Therfore, early surgical ligation may be an option to be considered rather than repetitive drug administration in these patients. The efficacy and side effect profiles of indomethacin, paracetamol, and ibuprofen were evaluated in a prospective study, conducted by El-Mashed et al. ${ }^{9}$ In that study, paracetamol was at least as effective as indomethacin and ibuprofen in PDA closure and had a lower risk, particularly in terms of renal function, platelet count, and gastrointestinal bleeding. In the study by Ko et al. including 41 VLBW premature infants, the direct surgical ligation was advocated to minimise PDA and recurrent medication complications in VLBW infants. ${ }^{1}$ In the study by Lee et al. in which 233 preterm infants with hsPDA below $1500 \mathrm{~g}$ were examined over nine years, early surgical intervention was found to be important for prevention of severe necrotising enterocolitis and severe intraventricular haemorrhage, facilitating early extubation in 134 patients, who underwent bedside surgical ligation. ${ }^{7}$ In the study by Avsar et al. which examined 26 VLBW infants, bedside ligation was a safe and effective method in PDA unresponsive to medical treatment. ${ }^{10}$ In the aforementioned study, there was no operation-related mortality; however, five patients died from sepsis, hypoxic encephalopathy, and respiratory failure during longterm follow-up.

Bedside PDA ligation can be performed by different surgical methods including posterior thoracotomy, anterior thoracotomy, median sternotomy, and limited upper sternotomy. The series of patients, who underwent PDA ligation with an anterior thoracotomy, in the study of Selcuk et al., mortality and complication rates during 10-year follow-up were found to be consistent with the literature. ${ }^{11}$ Similarly, in the study by Akyuz et al., conventional thoracotomy and upper limited sternotomy were compared; and no significant difference was found in terms of mortality rates. ${ }^{12}$

A properly organised NICU is required for bedside PDA closure. For this purpose, the NICU should be converted into an operating room when necessary. When the people's entrance the NICU are well-controlled, the necessary equipment (i.e, open bed, light source, cautery, and surgical set) is properly installed, and the organisation of the relevant teams (i.e, surgical team, neonatologist, surgical nurse, and support staff) takes place in time and, in accordance with the rules. This procedure does not cause any negative consequences for the patient. With proper organsation, time loss is expected to be less with reduced financial spending. ${ }^{13}$ Performing this operation at bedside in the NICU setting, shortens the duration of operation and anaesthesia withavoidance ofhypothermia. Extremecautionshould be practised while entering the thoracic cavity, and possible lung damage should be avoided. Besides, unnecessary retraction should be avoided during lung exclusion and the surgeon should avoid any damage to the already oedematous lung tissue.

In a retrospective study including newborns, who underwent PDA closure at the bedside in the operating room setting, bedside PDA closure did not increase the risk of surgical site 
infection or mortality, and haemodynamic problems were less in patients with bedside ligation. ${ }^{14}$ In the light of these data, bedside PDA closure is as effective as in-operation room PDA closure and a reliable method in experienced centres.

Although PDA ligation is typically a standard and uncomplicated procedure, patients are often small and immature, may have multiple comorbidities including blood pressure fluctuations, bleeding, respiratory compromise, infections, intraventricular haemorrhage, chylothorax, recurrent laryngeal nerve paralysis, and bronchopulmonary dysplasia, leading to even death. Preterm neonates treated with PDA ligation are also at risk for post-PDA ligation cardiac syndrome, characterised by hypotension requiring inotropic support and failure of ventilation and/or oxygenation 6 to 12 hours postoperatively. In this study, thanks to a well-planned organisation, all processes went smoothly. The active role of neonatologists at the intraoperative period is also vital for the maintenance of hemodynamic stability. No surgical or anaesthesia-related complications were observed. None of the patients developed post-PDA ligation syndrome. No surgical wound infection or sepsis was observed in any patient. Contrary to conventional beliefs, bedside ligation performed in the NICU setting did not increase the risk of surgical site infection and surgical site-associated sepsis. ${ }^{15}$ The overall mortality rate was also low (4.2\%).

In the current study, the patients were recruited in five different hospitals. As a common practice, although adult cardiac surgeries can be performed in two of these centres, paediatric cardiac surgeries are not available any of the study, centres. The avarage distance of the four other centres to this hospital was 5 to $15 \mathrm{~km}$. In this study, the authors attempted to prevent morbidity and mortality by transferring the surgical team to these centres ratherthan transferring the patients to this hospital, to avoidtransport-related complications of premature infants. Surgical equipment was provided by the surgical team before transfer of the surgical team.

\section{CONCLUSION}

On-site bedside PDA ligation at different NICUs by a roving surgical team is safe and effective, with an extremely low risk, in the hands of experienced surgeons. Surgical decision-making should not be delayed due to the negative consequences of hsPDA in premature infants. During the current novel coronavirus 2019 (COVID-19) outbreak, bedside PDA ligation may be reasonable to restrict patient transport between the centres. Nevertheless, further large-scale studies investigating the safety and efficacy of bedside PDA ligation are needed.

\section{ETHICALAPPROVAL:}

This study was approved by Ankara City Hospital Clinical Researches Ethics Committee (No. E1-20-1408, Date:23/12/2020).

\section{PATIENTS' CONSENT:}

A written informed consent was obtained prior to study from parents and/or legal guardians of the patients.

\section{CONFLICT OF INTEREST:}

The authors declared no conflict of interest.

\section{AUTHORS' CONTRIBUTION:}

BST: Acquisition, analysis.

AA: Drafting the work, revising.

DB: Analysis, interpretation.

EK: Analysis, final approval.

IT: Drafting the work.

\section{REFERENCES}

1. Ko YC, Chang Cl, Chiu IS, Chen YS, Huang SC, Hsieh WS. Surgical ligation of patent ductus arteriosus in very-lowbirth-weight premature infants in the neonatal intensive care unit. J Formos Med Assoc 2009; 108(1):69-71. doi: 10.1016/S0929-6646(09)60034-6.

2. Cassady G, Crouse DT, Kirklin JW, Strange MJ, Joiner CH, Godoy $\mathrm{G}$, et al. A randomized, controlled trial of very early prophylactic ligation of the ductus arteriosus in babies who weighed $1000 \mathrm{~g}$ or less at birth. N Engl J Med 1989; 320(23):1511-6. doi: 10.1056/NEJM198906083202302.

3. Cotton RB, Stahlman MT, Bender HW, Graham TP, Catterton WZ, Kovar I. Randomised trial of early closure of symptomatic patent ductus arteriosus in small preterm infants. J Pediatr 1978; 93(4):647-51. doi: 10.1016/s00223476(78)80910-x.

4. Yan H, Ma F, Li Y, Zhou K, Hua Y, Wan C. The optimal timing of surgical ligation of patent ductus arteriosus in preterm or very-low-birth-weight infants: A systematic review and meta-analysis. Medicine (Baltimore) 2020; 99(9):e19356. doi: 10.1097/MD.0000000000019356.

5. Weisz DE, Giesinger RE. Surgical management of a patent ductus arteriosus: Is this still an option? Semin Fetal Neonatal Med 2018; 23(4):255-266. doi: 10.1016/j.siny. 2018.03.003.

6. Hundscheid T, Onland W, van Overmeire B, Dijk P, van Kaam AHLC, Dijkman KP, et al. Early treatment versus expectative management of patent ductus arteriosus in preterm infants: A multicentre, randomised, non-inferiority trial in Europe (BeNeDuctus trial). BMC Pediatr 2018; 18(1):262. doi: 10.1186/s12887-018-1215-7.

7. Lee JH, Lee HJ, Park HK, Ahn JH, Kim HS, Jang HJ, et al. Surgical ligation of patent ductus arteriosus in preterm neonates weighing less than 1500g: A 9-year single center experience. J Cardiothorac Surg 2020; 15(1):144. doi: 10.1186/s13019-020-01191-2.

8. Trus T, Winthrop AL, Pipe S, Shah J, Langer JC, Lau GY. Optimal management of patent ductus arteriosus in the neonate weighing less than $800 \mathrm{~g}$. J Pediatr Surg 1993; 28(9):1137-9. doi: 10.1016/0022-3468(93)90148-e.

9. El-Mashad AE, El-Mahdy $H$, El Amrousy D, Elgendy M. Comparative study of the efficacy and safety of paracetamol, ibuprofen, and indomethacin in closure of patent ductus arteriosus in preterm neonates. Eur J Pediatr 2017; 176(2):233-240. doi: 10.1007/s00431-016-2830-7.

10. Avsar MK, Demir T, Celiksular C, Zeybek C. Bedside PDA ligation in premature infants less than 28 weeks and 1000 grams. J Cardiothorac Surg 2016; 11(1):146. doi: 10. 1186/s13019-016-0539-3.

11. Selcuk A, Cicek M, Yurdakok O, Kilic Y, İzgi Coskun F, Erdem $\mathrm{H}$, et al. Ligation of patent ductus arteriosus through 
anterior thoracotomy in preterm infants: A 10-year experience. Cardiol Young 2021; 28:1-7. doi: 10.1017/ S1047951121000032.

12. Akyuz M, Isik O, Mercan I, Cakmak M. Bedside surgical ligation of the patent ductus arteriosus in very-low-birthweight premature infants: Limited upper ministernotomy as an alternative approach. J Card Surg 2021; 36(2):436-41. doi: $10.1111 /$ jocs. 15171.

13. John T, Colvin R, Ferrall B. Improving the management and delivery of bedside patent ductus arteriosus ligation. AORN J 2007; 86(2):231-8. doi: 10.1016/j.aorn.2007.03.005.
14. Lee LK, Woodfin MY, Vadi MG, Grogan TR, Ross PJ, Applegate $\mathrm{RL}$, et al. A comparison of postoperative outcomes with PDA ligation in the OR versus the NICU: A retrospective cohort study on the risks of transport. $B M C$ Anesthesiol 2018; 18(1):199. doi: 10.1186/s12871018-0658-6.

15. Mirea L, Sankaran K, Seshia M, Ohlsson A, Allen AC, Aziz K, et al. Treatment of patent ductus arteriosus and neonatal mortality/morbidities: Adjustment for treatment selection bias. J Pediatr 2012; 161(4):689-94. doi: 10.1016/j.jpeds. 2012.05.007. 